\title{
Very large-scale neighborhoods with performance guarantees for minimizing makespan on parallel machines
}

Citation for published version (APA):

Brueggemann, T., Hurink, J. L., Vredeveld, T., \& Woeginger, G. J. (2006). Very large-scale neighborhoods with performance guarantees for minimizing makespan on parallel machines. METEOR, Maastricht University School of Business and Economics. METEOR Research Memorandum No. 052 https://doi.org/10.26481/umamet.2006052

Document status and date:

Published: 01/01/2006

DOI:

10.26481/umamet.2006052

Document Version:

Publisher's PDF, also known as Version of record

Please check the document version of this publication:

- A submitted manuscript is the version of the article upon submission and before peer-review. There can be important differences between the submitted version and the official published version of record.

People interested in the research are advised to contact the author for the final version of the publication, or visit the DOI to the publisher's website.

- The final author version and the galley proof are versions of the publication after peer review.

- The final published version features the final layout of the paper including the volume, issue and page numbers.

Link to publication

\footnotetext{
General rights rights.

- You may freely distribute the URL identifying the publication in the public portal. please follow below link for the End User Agreement:

www.umlib.nl/taverne-license

Take down policy

If you believe that this document breaches copyright please contact us at:

repository@maastrichtuniversity.nl

providing details and we will investigate your claim.
}

Copyright and moral rights for the publications made accessible in the public portal are retained by the authors and/or other copyright owners and it is a condition of accessing publications that users recognise and abide by the legal requirements associated with these

- Users may download and print one copy of any publication from the public portal for the purpose of private study or research.

- You may not further distribute the material or use it for any profit-making activity or commercial gain

If the publication is distributed under the terms of Article $25 \mathrm{fa}$ of the Dutch Copyright Act, indicated by the "Taverne" license above, 
Tobias Brueggemann, Johann L. Hurink, Tjark Vredeveld, Gerhard J. Woeginger

Very Large-Scale Neighborhoods with Performance Guarantees for Minimizing Makespan on Parallel Machines

$\mathrm{RM} / 06 / 052$

JEL code : C61

\section{METE@R}

Maastricht research school of Economics of TEchnology and ORganizations

Universiteit Maastricht

Faculty of Economics and Business Administration P.O. Box 616

NL - 6200 MD Maastricht

phone : + +31 433883830

fax : ++31433884873 



\section{Very Large-Scale Neighborhoods with Performance Guarantees for Minimizing Makespan on Parallel Machines}

Tobias Brueggemann ${ }^{1, *}$, Johann L. Hurink ${ }^{2}$,

Department of Applied Mathematics, University of Twente, P.O. Box 217, 7500 AE Enschede, The Netherlands

Tjark Vredeveld,

Department of Quantitative Economics, Maastricht University, P.O. Box 616, 6200 MD Maastricht, The Netherlands

\section{Gerhard J. Woeginger ${ }^{2}$.}

Dept. of Math. and Comp. Sci., Eindhoven University of Technology, P.O. Box 513, 5600 MB Eindhoven, The Netherlands

\section{Introduction}

In this paper, we consider the following multiprocessor scheduling problem. Given are $n$ jobs each of which has to be scheduled on one of $m$ identical parallel machines. The time it takes for a job to be fully processed is denoted by $p_{j}$. A machine can process at most one job at a time, and a job may not be preempted. The goal is to schedule the jobs in such a way that the makespan is minimized, i.e., we want the last job to complete as early as possible. In the standard notation of [8], this problem is denoted as $P \| C_{\max }$.

\footnotetext{
* Corresponding author.

Email addresses: t.brueggemann@math.utwente.nl (Tobias Brueggemann),

j.l.hurink@math.utwente.nl (Johann L. Hurink), t.vredeveld@ke.unimaas.nl (Tjark Vredeveld), gwoegi@win.tue.nl (Gerhard J. Woeginger).

1 supported by the Netherlands Organization for Scientific Research (NWO) grant 613.000.225 (Local Search with Exponential Neighborhoods).

2 supported by BSIK grant 03018 (BRICKS: Basic Research in Informatics for Creating the Knowledge Society).
} 
This problem is known to be strongly NP-hard for $m$ being part of the input [7]. Therefore, we search for approximate solutions. If an algorithm is guaranteed to deliver a solution that has value at most $\rho$ times the optimal solution value, we call it a $\rho$-approximation algorithm; the value $\rho$ is called the (worstcase) performance guarantee. A well known approximation algorithm for the problem under consideration is the LPT-rule due to Graham [9]: starting from an empty schedule, we select the jobs with longest processing time among the unscheduled jobs and schedule this job on the machine with currently minimal workload. This LPT-algorithm has a performance guarantee of $4 / 3-1 / 3 m$.

Another way to find approximate solutions is through local search, see e.g. [1]. These methods iteratively search through the set of feasible solutions. Starting from an initial solution, a local search procedure moves from one feasible solution to a neighboring one until some stopping criteria are met. The choice of a suitable neighborhood function has an important influence on the performance of local search.

The simplest form of local search is iterative improvement, also called local improvement or, in the case of minimization problems, descent algorithms. This method iteratively chooses a better solution in the neighborhood of the current one and it stops when no better solution is found. The final solution is called a local optimum.

Recently, there has been an increasing interest in the quality of local optima and the time needed to obtain these local optima through iterative improvement. For the parallel machine scheduling problem under consideration, Finn and Horowitz [6] showed that a so-called move-optimal solution is guaranteed to deliver a solution with value no more than $2-2 /(m+1)$ times the optimal makespan. Moreover, this bound is tight [11]. Brucker et al. [3] showed that the iterative improvement procedures needs $\mathcal{O}\left(n^{2}\right)$ moves to come to local optimal solution, and this bound is tight [10]. For performance guarantees of local search methods regarding makespan minimization, we refer to $[11,10]$. If the objective is to minimize total weighted completion time, Brueggemann et al. [4] gave a performance guarantee of $3 / 2-1 / 2 m$ for move-optimal schedules.

Over the last years, very large-scale neighborhoods has received considerable attention. These neighborhoods mostly contain up to an exponential number of solutions, but allow a polynomial exploration. A survey about very largescale neighborhood techniques is given by Ahuja et al. [2] and Derneko and Woeginger [5] present an overview of very large-scale neighborhoods for the traveling salesman and quadratic assignment problem.

In Section 2, we define a very large-scale neighborhood, called splitneighborhood and in the following sections we investigate its worst-case behavior. In Section 3, we see that a split-optimal solution has the same perfor- 
mance guarantee as the simple move-optimal solutions. In Sections 4 and 5 , we give performance guarantees on combined move- and split-optimal solutions. If we combine the two neighborhoods in the most straightforward way, we see that the performance guarantee marginally improves but is still essentially 2 , whereas a better combination leads to a performance guarantee of $3 / 2$.

\section{Neighborhoods}

As mentioned in the introduction, an important part of local search algorithms is the definition of the neighborhood on which the method operate. Before discussing the neighborhoods, we first describe our representation of a schedule. As the sequence in which the jobs are processed does not influence the makespan of a schedule for a given assignment, we represent a schedule by such an assignment of jobs to machines, $A: J \rightarrow\{1, \ldots, m\}$, where $J=\{1, \ldots, n\}$ is the set of jobs. This is equivalent to a partitioning of the set of jobs into $m$ disjoint subsets $M_{1}^{A}, \ldots, M_{m}^{A}$, where $M_{i}^{A}=\{j \in J: A(j)=i\}$ is the set of jobs scheduled on machine $i$. If there is no ambiguity, we write $M_{i}$ for $M_{i}^{A}$. The workload of machine $i$ is denoted by

$$
L_{i}^{A}=\sum_{j \in M_{i}} p_{j}
$$

and this workload is equal to the completion time of the last job scheduled on machine $i$. Again, if there is no ambiguity, we write $L_{i}$ for $L_{i}^{A}$. Hence, for a given assignment $A$ of jobs to machines, the makespan is equal to the machine with maximum workload:

$$
C_{\max }^{A}=\max _{i} L_{i}^{A} .
$$

We call such a machine with maximum workload a critical machine.

The move-neighborhood Probably the most basic neighborhood is the move-neighborhood. A neighbor is obtained by moving one job from its current machine to another machine. More formally, let $k$ be the selected job and $h$ be the selected machine. Then the neighbor obtained by moving job $k$ to machine $h$ is the assignment

$$
A_{\text {move }}^{(k, h)}(j)=\left\{\begin{array}{lll}
h & \text { if } & j=k \\
A(j) & \text { if } & j \neq k
\end{array}\right.
$$

Note that $A_{\text {move }}^{(k, h)}$ only differs from $A$ if $A(k) \neq h$. The neighborhood $\mathcal{N}_{\text {move }}(A)$ of an assignment $A$ contains all assignments that can be obtained by moving 

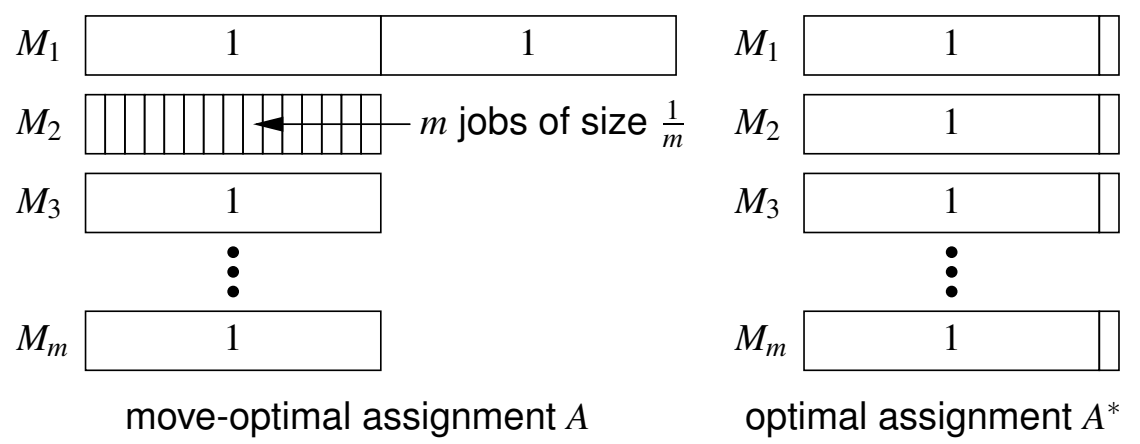

Fig. 1. Worst-case move-optimal schedule

one job to some machine, i.e.,

$$
\mathcal{N}_{\text {move }}(A)=\left\{A_{\text {move }}^{(k, h)}: k \in J \text { and } h=1, \ldots, m\right\} .
$$

We call an assignment $A$ move-optimal if for all $A^{\prime} \in \mathcal{N}_{\text {move }}(A), C_{\max }^{A} \leq C_{\max }^{A^{\prime}}$ and the number of critical machines in $A$ is at most the number of critical machines in $A^{\prime}$. Finn and Horowitz [6] gave the following upper bound on the performance guarantee of move-optimal schedules.

Theorem 1 Let $A$ be a move-optimal assignment, and let $C_{\max }^{*}$ denote the optimal makespan. Moreover, let $n_{k}=\max \left\{\left|M_{i}\right|: L_{i}=C_{\max }^{A}\right\}$, the (maximum) number of jobs on a critical machine in the schedule $A$. Then

$$
C_{\max }^{A} \leq \frac{n_{k} m}{\left(n_{k}-1\right) m+1} C_{\max }^{*}
$$

Moreover, if $n_{k}=1$, then $C_{\max }^{A}=C_{\max }^{*}$.

The bound in Theorem 1 attains its maximum for $n_{k}=2$, yielding a performance guarantee of $2-2 /(m+1)$. This bound has been proven tight by Schuurman and Vredeveld [11], see Figure 1 for the schedule attaining this bound.

The split-neighborhood The split-neighborhood is one of exponential size in the number of machines. To obtain a split-neighbor, we first partition the set of jobs assigned to machine $i$ into two disjoint sets. Hereto, we use a split-operator split : $2^{J} \rightarrow 2^{J} \times 2^{J}$. In the remaining, we assume w.l.o.g. that the split-operator on the set $M_{i}$ produces split $\left(M_{i}\right)=\left(M_{i 1}, M_{i 2}\right)$ and the partitioning is done so that

$$
L_{i 1}=\sum_{j \in M_{i 1}} p_{j} \geq \sum_{j \in M_{i 2}} p_{j}=L_{i 2} .
$$

This can always be achieved by simply exchanging the sets $M_{i 1}$ and $M_{i 2}$. We will refer to the set $M_{i 1}$ (resp. $M_{i 2}$ ) as the left (resp. right) part of $M_{i}$ or 
machine $i$.

By splitting all sets $M_{i}$ given by an assignment $A$, we obtain $2 m$ sets. Abusing notation, we denote the set of these $2 m$ sets by

$$
\operatorname{split}(A)=\left\{M_{i 1}, M_{i 2}: \operatorname{split}\left(M_{i}\right)=\left(M_{i 1}, M_{i 2}\right) \text { for } i=1, \ldots, m\right\} .
$$

The neighborhood $\mathcal{N}_{\text {split }}(A)$ of a schedule $A$ contains all schedules that can be obtained by assigning the jobs of exactly two of the $2 m$ sets from $\operatorname{split}(A)$ to a machine, i.e.,

$$
\begin{array}{r}
\mathcal{N}_{\text {split }}(A)=\left\{A^{\prime}=\left(M_{1}^{\prime}, \ldots, M_{m}^{\prime}\right): M_{i}^{\prime}=S_{i 1} \cup S_{i 2} \text { with } S_{i 1}, S_{i 2} \in \operatorname{split}(A),\right. \\
\left.S_{i 1} \cap S_{i 2}=\emptyset \text { and } M_{i}^{\prime} \cap M_{k}^{\prime}=\emptyset \text { for } i \neq k\right\}
\end{array}
$$

Assuming that each set $S \in \operatorname{split}(A)$ is non-empty, the number of neighbors for a schedule $A$ is equal to the number of perfect matching in a complete graph on $2 m$ vertices. Hence, in this case the size of the neighborhood is $|\mathcal{N}(A)|=\left(\begin{array}{c}2 m \\ m\end{array}\right) \frac{m !}{2^{m}} \geq\left(\frac{\sqrt{m}}{2}\right)^{m}$.

Although the size of the neighborhood is exponentially large in the number of machines, the following (folklore) fact tells us that the best neighbor can be found in $\mathcal{O}(m \log m)$ time.

Fact 2 Given $2 m$ numbers $a_{1} \geq \ldots \geq a_{2 m}$. A perfect matching of these numbers such that the maximum of the sum of the two numbers of a pair is minimized, is obtained by matching $a_{i}$ to $a_{2 m+1-i}$. Moreover, this matching minimizes the number of pairs whose sum equals this maximum.

We call an assignment split-optimal if for all $A^{\prime} \in \mathcal{N}_{\text {split }}(A), C_{\max }^{A} \leq C_{\max }^{A^{\prime}}$ and the number of critical machines in $A$ is at most the number of critical machines in $A^{\prime}$.

Combinations of move and split-neighborhood As we will see in the following section, a split-optimal schedule needs not to be a move-optimal schedule. Hence, we also consider move- and split-optimal schedules. The straightforward extension of this would be to define a move- and split-optimal schedule as one that is move-optimal as well as split-optimal. These local optima may however be improved by moving one job neither increasing nor decreasing the makespan, and then find a better split-neighbor. Therefore, we define a lexicographic-move-optimal schedule. For a given solution $A$ and $A^{\prime} \in \mathcal{N}_{\text {move }}(A)$, we reorder the machines in $A$ and $A^{\prime}$ so that

$$
\begin{aligned}
& L_{1}^{A} \geq \ldots \geq L_{m}^{A} \text { and } \\
& L_{1}^{A^{\prime}} \geq \ldots \geq L_{m}^{A^{\prime}} .
\end{aligned}
$$


The schedule $A^{\prime}$ is called lexicographically better than $A$, if there exists a machine $k$ such that

$$
\begin{aligned}
& L_{i}^{A^{\prime}}=L_{i}^{A} \quad \text { for } i=1, \ldots, k-1, \\
& L_{k}^{A^{\prime}}<L_{k}^{A} .
\end{aligned}
$$

We say that $A$ is lexicographical-move-optimal, or lexmove-optimal, if there exists no move-neighbor $A^{\prime} \in \mathcal{N}_{\text {move }}(A)$ that is lexicographically better than A.

Note that the move-optimal assignment $A$ in Figure 1 is also lexmove-optimal. Therefore, the move and the lexmove-optimal schedules have the same performance guarantee. On the other hand, the performance guarantee of a lexmove and split-optimal schedule is better than that of a move and split-optimal schedule.

\section{Performance guarantee on split-optimal schedules}

The quality of split-optimal assignments depends on the split-operator. In this paper, we consider only split-operators that obtain move-optimal partitions, i.e., if split $\left(M_{i}\right)=\left(M_{i 1}, M_{i 2}\right)$, with $L_{i 1} \geq L_{i 2}$, then for all $j \in M_{i 1}$, we have $L_{i 2}+p_{j} \geq L_{i 1}$. We call such a split-operator a move-optimal split-operator.

Due to Fact 2, we assume w.l.o.g. that the numbering of the machines is so that

$$
\begin{aligned}
& L_{i 1} \geq L_{i 2} \quad \text { for } i=1, \ldots, m \\
& L_{i 1} \geq L_{i+1,1} \text { for } i=1, \ldots, m-1 \\
& L_{i 2} \leq L_{i+1,2} \text { for } i=1, \ldots, m-1
\end{aligned}
$$

The performance guarantee of a split-optimal schedule, using a move-optimal split-operator, does not improve on the bound obtained by move-optimal schedules.

Theorem 3 Let $A$ be a split-optimal schedule using a move-optimal splitoperator. Then the makespan $A$ is bounded by $C_{\max }^{A} \leq\left(2-\frac{2}{m+1}\right) C_{\max }^{*}$, where $C_{\max }^{*}$ denotes the value of the optimal makespan.

PROOF. W.l.o.g. we assume that $C_{\max }^{A}=1$. Let $k$ be a critical machine in the schedule $A$. If $\sum_{j} p_{j} \geq m L_{k 1}+L_{k 2}$, then the optimal makespan can be bounded from below by $C_{\max }^{*} \geq \frac{1}{m} \sum_{j} p_{j} \geq L_{k 1}+L_{k 2} / m$. Using the fact that 
$L_{k 1}+L_{k 2}=1$, we have

$$
\frac{C_{\max }^{A}}{C_{\max }^{*}} \leq \frac{m}{(m-1) L_{k 1}+1} \leq \frac{2 m}{m+1},
$$

as the above expression is maximized for minimal $L_{k 1}$ and by $L_{k 1} \geq L_{k 2}$ we know that $L_{k 1} \geq 1 / 2$.

On the other hand, if $\sum_{j} p_{j}<m L_{k 1}+L_{k 2}$, then a machine $l$ with minimal load satisfies

$$
L_{l} \leq \sum_{i \neq k} L_{i} /(m-1)<L_{k 1} .
$$

Moreover, by (2), we know that (3) implies $L_{l 2} \geq L_{k 2}$. Hence,

$$
L_{k 1}>L_{l} \geq 2 L_{l 2} \geq 2 L_{k 2} \text {. }
$$

From the fact that a move-optimal split-operator is used to obtain the sets $M_{i 1}$ and $M_{i 2}$, we know that for all $j \in M_{k 1}, L_{k 2}+p_{j} \geq L_{k 1}$. Therefore, from (4) it follows that $p_{j}>\frac{1}{2} L_{k 1}$ for all $j \in M_{i 1}$. Hence, $M_{i 1}$ contains only one job and

$$
C_{\max }^{A}=L_{k}=L_{k 1}+L_{k 2}<\frac{3}{2} L_{k 1} \leq \frac{3}{2} C_{\max }^{*},
$$

as $C_{\max }^{*} \geq p_{j}$ for all $j \in J$ and thus $C_{\max }^{*} \geq L_{k 1}$.

For $m \geq 3$, the theorem is proven, as $\frac{3}{2} \leq 2 m /(m+1)$. For $m=2$, it follows from (4) that

$$
C_{\max }^{*} \geq \frac{1}{2} \sum_{j} p_{j} \geq \frac{1}{2}\left(L_{k}+2 L_{l 2}\right) \geq \frac{1}{2}\left(L_{k 1}+3 L_{k 2}\right)=\frac{3}{2}-L_{k 1},
$$

where the last equality follows from the fact that $L_{k 2}=1-L_{k 1}$. Moreover, as $C_{\max }^{*} \geq L_{k 1}$, due to the fact that $M_{k 1}$ contains only one job, we have

$$
C_{\max }^{*} \geq \max \left\{L_{k 1}, \frac{3}{2}-L_{k 1}\right\}
$$

which is minimal for $L_{k 1}=\frac{3}{4}$. Therefore, for $m=2$, we have

$$
C_{\max }^{A} \leq \frac{4}{3} C_{\max }^{*}
$$

To show that the analysis is tight, consider the folowing instance consisting of $m$ jobs with processing time 1 and $m$ jobs with processing time $1 / m$. In the split-optimal assignment $A$, we schedule on every machine one job with processing time 1 and on the first machine all jobs with processing time $1 / \mathrm{m}$ are scheduled. It is easy to check that this schedule is split-optimal for a 

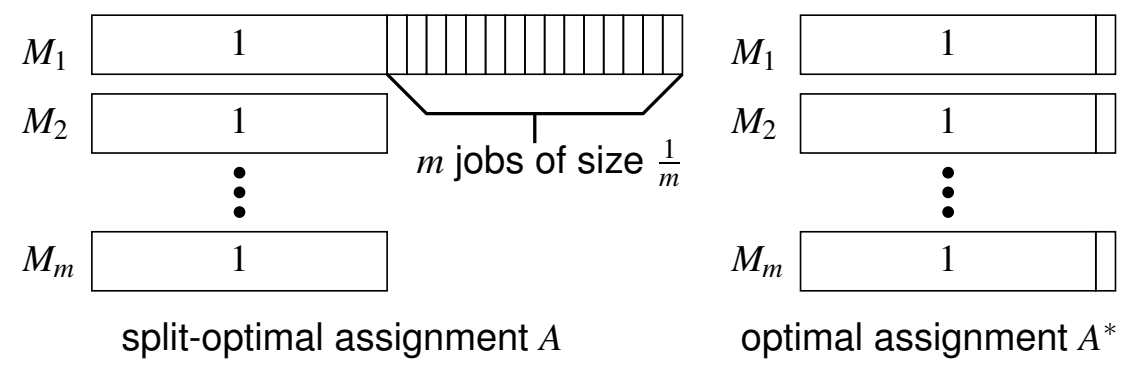

Fig. 2. Split optimal assignment $A$ and optimal schedule $A^{*}$

move-optimal split-operator and it has makespan $C_{\max }^{A}=2$. In an optimal assignment $A^{*}$, we schedule on every machine one job with processing time 1 and one with processing time $1 / \mathrm{m}$. The optimal makespan is $C_{\max }^{*}=1+1 / \mathrm{m}$, and thus $C_{\max }^{A}=2 m /(m+1) C_{\max }^{*}$. See Figure 2 for an illustration.

\section{Split-optimal and move-optimal schedules}

The split-optimal schedule, showing the tightness of our analysis in the previous section, is obviously not move-optimal. This raises the question whether a combination of the two neighborhoods gives a better performance guarantee, which is answered in Theorem 9, for move-optimal split-operators. Moreover, if the total processing time of the jobs compared to the load of the left part of a critical machine is small, we can even prove that this move- and split-optimal schedule is globally optimal.

Like in the previous section, we assume w.l.o.g. that for a local optimal schedule, $A$, property (2) holds, and that $C_{\max }^{A}=1$.

Lemma 4 Let $A$ be a move-and split-optimal schedule using a move-optimal split-operator. If there exists a critical machine $k$ with

$$
\sum_{j} p_{j}<m L_{k 1}+L_{k 2}
$$

then $C_{\max }^{A}=C_{\max }^{*}$, where $C_{\max }^{*}$ denotes the optimal makespan.

PROOF. Let $l$ be a machine with minimal load. Then, we know that $L_{l}<$ $L_{k 1}$. By move-optimality of the schedule $A$, we know that for any job $j \in M_{k}$

$$
L_{l}+p_{j} \geq L_{k}=L_{k 1}+L_{k 2} .
$$

Hence, $p_{j}>L_{k 2}$ for $j \in M_{k}$, and thus $M_{k 2}$ contains no job at all, i.e., $L_{k 2}=0$. It follows from the move-optimal split-operator, that whenever $M_{k 2}$ is empty, $M_{k 1}$ contains only one job, $j_{1}$. Hence, $C_{\max }^{A}=L_{k}=p_{j_{1}} \leq C_{\max }^{*}$. 
From this lemma, it follows that we only have to consider cases in which the total load on all machines is large enough. Moreover, if $L_{k 1}$ is large enough, we can actually prove a bound on the makespan of this local optimal schedule, which is better than the guarantee in Theorem 9 .

Lemma 5 Let $A$ be a move- and split-optimal schedule obtained by using a move-optimal split-operator. If there exists a critical machine $k$, satisfying $L_{k 1} \geq \frac{2}{3}$, then the makespan of $A$ can be bounded by

$$
C_{\max }^{A} \leq \frac{3 m}{2 m+1} C_{\max }^{*}
$$

where $C_{\max }^{*}$ denotes the optimal makespan.

PROOF. By Lemma 4, we only have to consider the case that $\sum_{j} p_{j} \geq$ $m L_{k 1}+L_{k 2}$. Hence, the optimal makespan can be bounded from below by $C_{\max }^{*} \geq \frac{1}{m} \sum_{j} p_{j} \geq \frac{(m-1) L_{k 1}+1}{m}$. As, $C_{\max }^{A}=1$, we thus have

$$
\frac{C_{\max }^{A}}{C_{\max }^{*}} \leq \frac{m}{(m-1) L_{k 1}+1} \leq \frac{3 m}{2 m+1}
$$

where the last inequality is due to $L_{k 1} \geq 2 / 3$.

Before we prove the performance guarantee of a move- and split-optimal schedule $A$, we first partition the set of machines into several subsets, based on the properties of the machines in such a move- and split-optimal schedule.

$$
\begin{aligned}
& S_{<}=\left\{i: L_{i 1}<L_{k 1}\right\}, \\
& S_{\geq}=\left\{i: L_{i 1} \geq L_{k 1}\right\}, \\
& S_{\text {multi }}=\left\{i \in S_{\geq}:\left|M_{i 1}\right| \geq 2\right\}, \\
& S_{\text {single }}=S_{\geq} \backslash\left(S_{\text {multi }} \cup\{k\}\right) .
\end{aligned}
$$

That is, $S_{<}$is the set of machines that have a left part which is smaller than $L_{k 1}$, and $S_{\geq}$is the set of the remaining machines. This set is again partitioned in one set containing all machines that have at least two jobs in the left part and the remaining machines in $S_{\geq} \backslash\{k\}$ containing exactly one job in the left part Note that, $S_{\geq} \backslash\{k\}=S_{\text {multi }} \cup S_{\text {single }}$.

The load of a machine in each of the above classes, we can bound as follows.

Lemma 6 Let $A$ be a move- and split-optimal schedule, for a move-optimal split-operator and let $k$ be a critical machine in this schedule. Moreover, let 
$S_{<}$and $S_{m u l t i}$ be as defined in (5). Then

$$
\begin{array}{ll}
L_{i} \geq 2\left(1-L_{k 1}\right) & \text { for } \quad i \in S_{<} \\
L_{i} \geq \frac{3}{2} L_{k 1} & \text { for } \quad i \in S_{\text {mult } i} .
\end{array}
$$

PROOF. Consider a machine $i \in S_{<}$. Then by property (2), we know that $L_{i 1}<L_{k 1}$ implies that $L_{i 2} \geq L_{k 2}$. Moverover, as $L_{i 1} \geq L_{i 2}$, we have that $L_{i} \geq 2 L_{i 2} \geq 2 L_{k 2}=2\left(1-L_{k 1}\right)$.

Now, let $i \in S_{\text {multi }}$, and let $j_{s} \in M_{i 1}$ be the smallest job in the left part of machine $i$. As $M_{i 1}$ contains at least two jobs, we know that $p_{j_{s}} \leq \frac{1}{2} L_{i 1}$. Due to the move-optimality of the split-operator, we also know that $L_{i 2} \geq L_{i 1}-p_{j_{s}} \geq$ $\frac{1}{2} L_{i 1}$. Hence, $L_{i} \geq \frac{3}{2} L_{i 1} \geq \frac{3}{2} L_{k 1}$.

Lemma 7 Let $A$ be a move- and split-optimal schedule for a move-optimal split-operator and $k$ be a critical machine in $A$. Moreover, let $S_{<}$be as defined in (5). If $1 / 2 \leq L_{k 1} \leq 2 / 3$ and $\left|S_{<}\right| \geq 1$, then

$$
\frac{C_{\max }^{A}}{C_{\max }^{*}} \leq \begin{cases}\frac{2 m}{m+2} & \text { for } m \geq 4 \\ \frac{3 m}{2 m+1} & \text { for } m \leq 3\end{cases}
$$

where $C_{\max }^{*}$ denotes the optimal makespan.

PROOF. Using Lemma 6, we can bound the optimal makespan by

$$
\begin{aligned}
m C_{\max }^{*} & \geq \sum_{j} p_{j} \geq 1+2\left|S_{<}\right|\left(1-L_{k 1}\right)+\left(m-1-\left|S_{<}\right|\right) L_{k 1} \\
& \geq 1+\left(2-3 L_{k 1}\right)\left|S_{<}\right|+(m-1) L_{k 1} \geq 3+(m-4) L_{k 1},
\end{aligned}
$$

where the last inequality is due to $L_{k 1} \leq 2 / 3$. For $m \geq 4$, the expression in (6) is minimized for $L_{k 1}$ minimal, whereas for $m \leq 3$, it is minimized for $L_{k 1}$ maximal. Using the fact that $1 / 2 \leq L_{k 1} \leq 2 / 3$ and $C_{\max }^{A}=1$, we have

$$
\frac{C_{\max }^{A}}{C_{\max }^{*}} \leq \begin{cases}\frac{2 m}{m+2} & \text { for } m \geq 4 \\ \frac{3 m}{2 m+1} & \text { for } m \leq 3\end{cases}
$$

Lemma 8 Let $A$ be a move-and split-optimal schedule for a move-optimal split-operator and $k$ be a critical machine in $A$. Moreover, let $S_{\text {multi }}$ be as 
defined in (5). If $1 / 2 \leq L_{k 1} \leq 2 / 3$ and $\left|S_{\text {multi }}\right| \geq 2$, then

$$
\frac{C_{\max }^{A}}{C_{\max }^{*}} \leq \frac{2 m}{2 m+2}
$$

where $C_{\max }^{*}$ denotes the optimal makespan.

PROOF. Consider a move- and split-optimal schedule $A$ for a move-optimal split-operator and let $S_{<}, S_{\text {multi }}$, and $S_{\text {single }}$ be as defined in (5). For $L_{k 1} \leq 2 / 3$, we know from Lemma 6 that for $i \in S_{<}, L_{i} \geq L_{k 1}$. Hence, using Lemma 6 , we can bound the optimal makespan by

$$
C_{\max }^{*} \geq \frac{1+\left(\left|S_{\mathrm{multi}}\right| / 2+m-1\right) L_{k 1}}{m} \geq \frac{1+m L_{k 1}}{m} \geq \frac{2+m}{2 m}
$$

where the second inequality is due to $\left|S_{\text {multi }}\right| \geq 2$ and the last is due to $L_{k 1} \geq 1 / 2$. As by assumption $C_{\max }^{A}=1$, the lemma is proven.

Theorem 9 A move-and split-optimal schedule, obtained by a move-optimal split-operator, has a performance guarantee of $2-\frac{4}{m+3}$.

PROOF. Let $A$ be a move- and split-optimal schedule for a move-optimal split-operator and let $k$ be a critical machine in $A$. Because $\frac{3 m}{2 m+1} \leq \frac{2 m+2}{m+3}$, by Theorem 1 we only need to consider a move- and split-optimal schedule $A$ in which a critical machine $k$ contains exactly two jobs. Morever, by Lemma 5 , we may restrict ourselves to the case that $L_{k 1}=\in\left[\frac{1}{2}, \frac{2}{3}\right]$, and by Lemma 4 , we may assume that $\sum_{j} p_{j} \geq m L_{k 1}+L_{k 2}$.

As $\frac{2 m}{m+2} \leq \frac{2 m+2}{m+3}$, we can restrict ourselves to the case that there is no machine $i$ with $L_{i 1}<L_{k 1}$ and at most one machine $i$ with $\left|M_{i 1}\right| \geq 2$. Note that if no such machine exists, there are $m$ jobs of length at least $L_{k 1}$ and one job of length $1-L_{k 1} \leq L_{k 1}$. Then, by the pigeonhole principle $C_{\max }^{*}=C_{\max }^{A}$. Hence, we assume that there is exactly one machine $s$ with $M_{s 1} \geq 2$.

Let $j_{1}$ be the smallest job in $M_{s 1}$. If $p_{j_{1}} \geq \frac{m+3}{2 m+2}-L_{k 2}$, then there are $m-1$ jobs of length $L_{k 1}$, one job of length $L_{k 2}=1-L_{k 1} \leq L_{k 1}$ and at least two jobs of length $\frac{m+3}{2 m+2}-L_{k 2}$, and by the pigeonhole principle, we know that $C_{\max }^{*} \geq \frac{m+3}{2 m+2}$.

On the other hand, if $p_{j_{1}} \leq \frac{m+3}{2 m+2}-\left(1-L_{k 1}\right)$, we can bound the load of the right part of machine $s$ by $L_{s 2} \geq L_{s 1}-p_{j_{1}}$ Hence, using the fact that $L_{k 1} \geq 1 / 2$, we 
can bound the total workload by

$$
\begin{aligned}
\sum_{j} p_{j} & \geq L_{s}+\sum_{i \neq s} L_{i} \geq(m-1) L_{k 1}+1-L_{k 1}+L_{s 1}+L_{s 2} \\
& \geq(m-2) L_{k 1}+1+2 L_{s 1}-p_{j_{1}} \geq m L_{k 1}+1-p_{j_{1}} \\
& \geq(m-1) L_{k 1}+2-\frac{m+3}{2 m+2} \geq \frac{m-1}{2}+2-\frac{m+3}{2 m+2} \\
& =\frac{m^{2}+3 m}{2 m+2} .
\end{aligned}
$$

This implies that the optimal makespan can be bounded by

$$
C_{\max }^{*} \geq \frac{1}{m} \sum_{j} p_{j} \geq \frac{m+3}{2 m+2},
$$

and thus we obtain

$$
\frac{C_{\max }^{A}}{C_{\max }^{*}} \leq \frac{2 m+2}{m+3}
$$

For instances with an odd number of machines, the analysis of the previous theorem is tight. If we schedule $m$ jobs of length 1 and $m$ jobs of length $2 /(m+1)$ as illustrated by the assignment $A$ in Figure 3 , we obtain a moveand split-optimal schedule for a move-optimal split-operator, with makespan $C_{\max }^{A}=2$. In the optimal schedule, all machines have the same workload and $C_{\max }^{*}=1+\frac{2}{m+3}$. For the split-optimallity of this example, we need that the

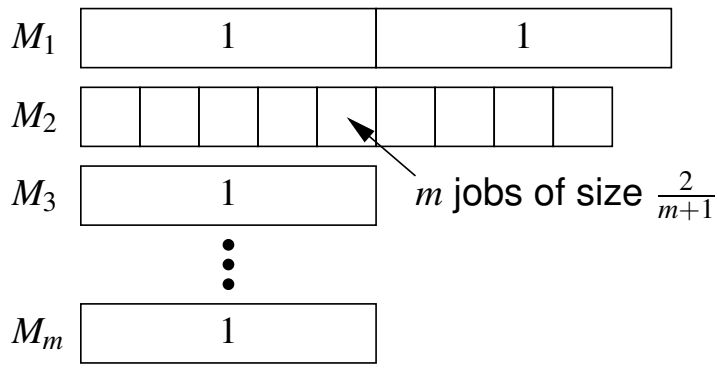

split- and move-optimal assignment $A$

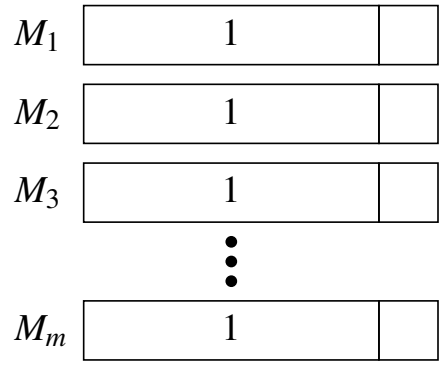

optimal assignment $A^{*}$

Fig. 3. Move- and split-optimal assignment and optimal schedule $A^{*}$

left part of machine $M_{2}$ has workload equal to 1 . Therefore, this example only works for an odd number of machines. For even number of machines, a lower bound on the performance guarantee is $\frac{2 m}{m+2}$. This bound is obtained by an instance with $m$ jobs of size 1 and $m-1$ jobs of size $2 / m$. In the move- and split-optimal schedule, these jobs are scheduled simular as in Figure 3.

In the case of $m=2$ machines, the bound cannot be improved either, as is shown by the example in Figure 4, which has makespan $C_{\max }^{A}=6$ for the local optimum and $C_{\max }^{*}=5$, in an optimal schedule. 

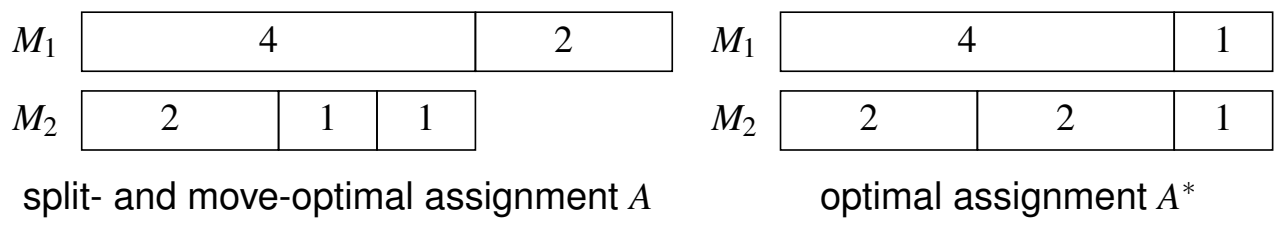

Fig. 4. Move- and split-optimal schedule for $m=2$.

\section{Split-optimal and lexicographic-move-optimal schedules}

In the previous section, we have seen that the performance guarantee of a move- and split-optimal schedule marginally improves on the performance guarantee of only a move- or only a split-optimal schedule. Moreover, the example, showing the tightness of the guarantee for an odd number of machines, is not lexicographic-move-optimal. Therefore, in this section we consider the lexmove- and split-optimal schedules.

For lexmove-optimal schedules, we have the following fact.

Fact 10 Let $A$ be an assignment of the jobs to the machines and let $l$ be a machine with minimal workload. A schedule represented by $A$ is lexmoveoptimal if and only if, for all machines $i$ and all jobs $j \in M_{i}$

$$
L_{l}+p_{j} \geq L_{i}
$$

In this section, we only consider the LPT rule as the split-operator. Remember that the LPT rule sorts the jobs in non-increasing size and then iteratively assigns a job to the set with minimal workload. In this way, we obtain a partition $\operatorname{LPT}\left(M_{i}\right)=\left(M_{i 1}, M_{i 2}\right)$ that is move-optimal. Therefore, we can apply Lemma $4-8$.

In the remainder of this section, we again assume w.l.o.g. that for a lexmoveand split-optimal schedule $A, C_{\max }^{A}=1$ and that (2) holds. Moreover, we also classify the machines into the sets $S_{<}, S_{\text {multi }}$, and $S_{\text {single }}$ as in (5).

Lemma 11 Let $A$ be a lexmove-and split-optimal schedule for a move-optimal split-operator. Let $k$ be a critical machine and $l$ be a machine with minimal load. Moreover, let $C_{\max }^{*}$ denote the optimal makespan. Then, if $l \in S_{<} \cup S_{\text {multi }}$,

$$
\frac{C_{\max }^{A}}{C_{\max }^{*}} \leq \frac{3 m}{2 m+1}
$$

PROOF. By Lemmas 4 and 5, we can restrict ourselves to the case that $\sum_{j} p_{j} \geq m L_{k 1}+L_{k 2}$ and $1 / 2 \leq L_{k 1} \leq 2 / 3$. 
If $l \in S_{<}$, we know from Lemma 6 that $L_{l} \geq 2\left(1-L_{k 1}\right) \geq 2 / 3$ and if $l \in S_{\text {multi }}$, it follows from Lemma 6 that $L_{l} \geq \frac{3}{2} L_{k 1} \geq 3 / 4 \geq 2 / 3$. Hence, from $L_{i} \geq L_{l}$, we have

$$
C_{\max }^{*} \geq \frac{1}{m}\left(1+\frac{2(m-1)}{3}\right)=\frac{2 m+1}{3 m} .
$$

By this lemma, we know that in order to prove the performance guarantee of $3 / 2$ in Theorem 13, we can restrict ourselves to local optimal schedules with $l \in S_{\text {single. }}$. Moreover, as $L_{l} \geq 2 / 3$ implies that $C_{\max }^{*} \geq 2 / 3$, we assume from here on that $L_{l}<2 / 3$.

In the proof of Theorem 13, we use the concept of blocking jobs.

Definition 12 A call a job $j$ a blocking job, if $p_{j}+L_{k 1} \geq 2 / 3$, where $L_{k 1}$ is the load of the left part of a critical machine.

Note that if, in some schedule, a blocking job is assigned to the same machine as a job of size at least $L_{k 1}$, then the makespan of this schedule will be at least $2 / 3$.

Theorem 13 Let $A$ be a lexmove- and split-optimal schedule, where the splitoperator is the LPT rule. Then,

$$
C_{\max }^{A} \leq \frac{3}{2} C_{\max }^{*}
$$

where, $C_{\max }^{*}$ denotes the optimal makespan.

PROOF. Let $A$ be a lexmove- and split-optimal schedule and let $k$ be a critical machine and $l$ a machine with minimal load. By Theorem 1 we may assume w.l.o.g. that $\left|M_{k}\right|=2$. Moreover, by Lemma 4 and 5, we restrict ourselves to the case that $\sum_{j} p_{j} \geq m L_{k 1}+L_{k 2}$ and $L_{k 1} \leq 2 / 3$. Finally, we define the sets $S_{<}, S_{\text {multi }}$, and $S_{\text {single }}$ as in (5). Then, by Lemma 11 we assume that $l \in S_{\text {single }}$ and $L_{l}<2 / 3$.

Under these assumptions, we claim that the total processing time of blocking jobs, scheduled on a machine $i \in S_{<} \cup S_{\text {multi }}$ is at least $2 / 3$. If in an optimal schedule at least one blocking job is scheduled on the same machine as a job of size at least $L_{k 1}$, then by definition of blocking jobs, this schedule has makespan at least $2 / 3$. On the other hand, if none of the blocking jobs, which $A$ assigns to a machine in $S_{<} \cup S_{\text {multi }}$, is scheduled together with a job of size at least $L_{k 1}$, then all these jobs need to be distributed over $\left|S_{<} \cup S_{\text {multi }}\right|$ machines, as each machine in $S_{\text {single }} \cup\{k\}$ processes at least one job with processing time at least $L_{k 1}$. From our claim, it now follows that the machine with maximal 
load from these blocking jobs has load at least $2 / 3$. Hence, in both cases we have $C_{\max }^{*} \geq 2 / 3$, and the theorem is proven.

To prove our claim, first consider a job $i \in S_{<}$. From Lemma 6 and lexmoveoptimality of A, it follows that a job $j \in M_{i}$ has processing time $p_{j} \geq L_{i}-L_{l} \geq$ $4 / 3-2 L_{k 1}$. Hence, $p_{j}+L_{k 1} \geq 4 / 3-L_{k 1} \geq 2 / 3$, as $L_{k 1} \leq 2 / 3$, and $j$ is a blocking job. As each job $j \in M_{i}$ is a blocking job, the total load of blocking jobs scheduled on machine $i \in S_{<}$is $L_{i} \geq 2\left(1-L_{k 1}\right) \geq 2 / 3$.

Now, consider a machine $i \in S_{\text {multi }}$, with $\left|M_{i 1}\right| \geq 3$. The smallest job in the left part, say $j_{0} \in M_{i 1}$ has length at most $p_{j_{0}} \leq L_{i 1} /\left|M_{i 1}\right|$. By move-optimality of the split-operator, we know that the load of the right part can be bounded by

$$
L_{i 2} \geq L_{i 1}-p_{j_{0}} \geq \frac{\left|M_{i 1}\right|-1}{\left|M_{i 1}\right|} L_{i 1} \geq \frac{2}{3} L_{i 1}
$$

Hence, by lexmove-optimality of the schedule, we know that any job $j \in M_{i}$ has processing time $p_{j} \geq L_{i}-L_{l} \geq \frac{5}{3} L_{i 1}-2 / 3$. Thus $p_{j}+L_{k 1} \geq \frac{8}{3} L_{k 1}-2 / 3 \geq 2 / 3$. Hence, each job $j \in\left\{i \in S_{\text {multi }}:\left|M_{i 1}\right| \geq 3\right\}$ is a blocking job, and the total processing times of the blocking jobs assigned to such a machine $i$ is $L_{i} \geq 2 / 3$.

Finally, consider a machine $i \in S_{\text {multi }}$, with $\left|M_{i 1}\right|=2$, say $M_{i 1}=\left\{j_{1}, j_{2}\right\}$ with $p_{j_{1}} \geq p_{j_{2}}$. By move-optimality of the split-operator, we know that $L_{i 2} \geq p_{j_{1}}$, and by move-optimality of the schedule $A$, we also know that $L_{l} \geq L_{i}-p_{j_{2}}=$ $L_{i 2}+p_{j_{1}} \geq 2 p_{j_{1}}$. Hence, $p_{j_{1}} \leq L_{l} / 2 \leq 1 / 3$.

This implies that $p_{j_{2}}=L_{i 1}-p_{j_{1}} \geq L_{k 1}-1 / 3 \geq 1 / 6$ and $p_{j_{2}}$ is a blocking job, as $L_{k 1}+1 / 6 \geq 2 / 3$. Moreover, due to the fact that the LPT-rule is used as a split operator, we know that there exists at least one job $j \in M_{i 2}$ in the right part of machine $i$ with $p_{j} \geq p_{j_{2}}$. Hence, $M_{i}$ contains at least three blocking jobs, $j_{1}, j_{2}$, and $j_{3}$, and the total processing time of these three jobs is at least

$$
p_{j_{1}}+p_{j_{2}}+p_{j_{3}} \geq L_{k 1}+1 / 6 \geq 2 / 3
$$

which completes the proof.

To show a lower bound on the performance guarantee, let $\delta=\frac{1}{3 m-4}$ and consider the instance consisting of $2 m-2$ jobs with processing time $3 \delta$, one job of size $1+\delta$ and $m-1$ jobs of length $2-\delta$. The schedule $A$ as depicted in Figure 5 is lexmove- and split-optimal and has makespan $C_{\max }^{A}=3$, whereas the optimal makespan is $C_{\max }^{*}=2+2 \delta$. This yields a ratio of

$$
\frac{C_{\max }^{A}}{C_{\max }^{*}}=\frac{3 m-4}{2 m-2}=\frac{3}{2}-\frac{1}{2 m-2} .
$$




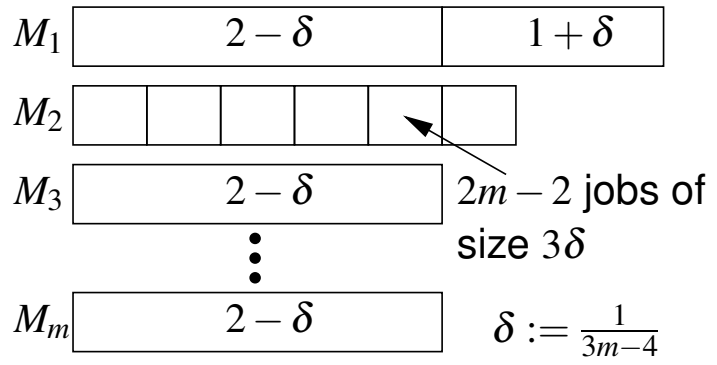

split- and lexmove-optimal assignment $A$

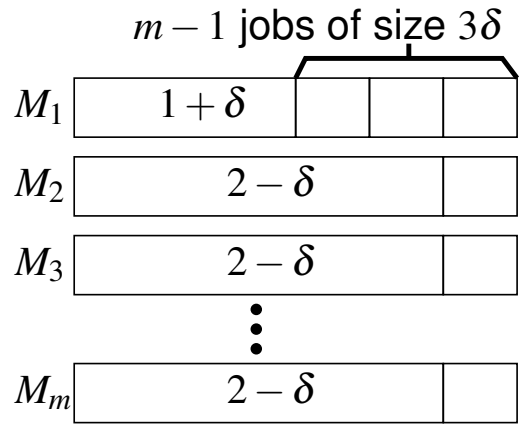

optimal assignment $A^{*}$

Fig. 5. A lexmove- and split-optimal schedule $A$

\section{References}

[1] E.H.L. Aarts and J.K. Lenstra (eds.), Local search in combinatorial optimization, John Wiley \& Sons, Chichester, UK, 1997.

[2] R.K. Ahuja, E. Özlem, J.B. Orlin, and A.P. Punnen, A survey of very largescale neighborhood search techniques, Discrete Applied Mathematics 123 (2002), 75-102.

[3] P. Brucker, J. Hurink, and F. Werner, Improving local search heuristics for some scheduling problems II, Discrete Applied Mathematics 72 (1997), 47-69.

[4] T. Brueggemann, J.L. Hurink, and W. Kern, Quality of move-optimal schedules for minimizing total weighted completion time, Operations Research Letters (2006), to appear.

[5] V.G. Deĭneko and G.J. Woeginger, A study of exponential neighborhoods for the travelling salesman problem and for the quadratic assignment problem, Mathematical Programming, Series A 87 (2000), 519-542.

[6] G. Finn and E. Horowitz, A linear time approximation algorithm for multiprocessor scheduling, BIT 19 (1979), 312-320.

[7] M.R. Garey and D.S. Johnson, Complexity results for multiprocessor scheduling under resource constraints, SIAM Journal on Computing 4 (1975), 397-411.

[8] R. L. Graham, E. L. Lawler, J. K. Lenstra, and A. H. G. Rinnooy Kan, Optimization and approximation in deterministic sequencing and scheduling: a survey, Annals of Discrete Mathematics 5 (1979), 287-326.

[9] R.L. Graham, Bounds on multiprocessing anomalies, SIAM Journal on Applied Mathematics 17 (1969), 416-429.

[10] C.A.J. Hurkens and T. Vredeveld, Local search for multiprocessor scheduling: How many moves does it take to a local optimum?, Operations Research Letters 31 (2003), 137-141. 
[11] P. Schuurman and T. Vredeveld, Performance guarantees of local search for multiprocessor scheduling, Informs Journal on Computing (2006), to appear. 